1193

\section{NOTING THE NEED FOR NEONATAL ONLINE TRAINING IN EUROPE - LEONARDO DA VINCI THE THIRD}

C. Peters ${ }^{1}$, S. Smith ${ }^{2}$, H. Budge ${ }^{3}$, J. Gadzinowski ${ }^{4}$, G. Griesen ${ }^{5}$, D. Neubauer ${ }^{6}$, E. Reiger-Fackeldey ${ }^{7}$, V. Heili ${ }^{8}$, M. Hall ${ }^{1}$

${ }^{1}$ Department of Neonatal Medicine, Southampton University Hospitals NHS Trust, ${ }^{2}$ School of Nursing and Midwifery, University of Southampton, Southampton, ${ }^{3}$ Academic Division of Child Health, University of Nottingham, Nottingham, UK, ${ }^{4}$ Department of Neonatology, University of Medical Sciences, Poznan, Poland, ${ }^{5}$ Department of Neonatology, Copenhagen University Hospital, Copenhagen, Denmark, ${ }^{6}$ Pediatrics, University Medical Center Ljubljana, Ljubljana, Slovenia,

${ }^{7}$ Department of Neonatology, University of Freiburg, Freiburg, Germany, ${ }^{8}$ Department of Neonatology, University Childrens Hospital, Tartu, Estonia

A European syllabus for postgraduate training in neonatology was published in 1998 but within Europe there remains a wide variation in the quality and standards of training programmes. In two previous projects we have demonstrated that online neonatal training modules can provide education and training of a uniform standard, which is accessible to all trainees across Europe, irrespective of geographical or personal circumstances.

A third online training programme (N.O.T.E.), funded by the European Commission Leonardo da Vinci Programme, has been developed to offer four, syllabus-based, training modules. Summative assessment will be integrated, with the aim of offering successful participants an accredited, transferable qualification. This paper describes the outcome of the recruitment process.

Methods: Details of the programme were circulated by the 7 European partners to colleagues and trainees in their own and neighbouring countries.

Results: Application forms, including a standardised questionnaire, were submitted by 197 doctors from 20 countries. $66 \%$ were female; the mean age of both sexes was 35 years, ranging from 25 to 58 years with up to 25 years neonatal experience reported.

Reasons for applying were; $86 \%$ wished to increase knowledge, $54 \%$ expected to gain from multicultural collaboration, $30 \%$ wanted to experience a new approach to learning. $62 \%$ had no prior online learning experience. The benefits of flexible studying and improved access to training resources were highlighted.

We conclude that there is demand for both online postgraduate education and continuing professional development in neonatal medicine to meet the needs of a diverse group of European doctors.

\section{4}

VLBW INFANTS BORN SMALL FOR DATES: INCIDENCE, RISK FACTORS AND OUTCOME

\author{
F.K. Chiou, S.K.Y. Ho \\ Department of Neonatal and Developmental \\ Medicine, Singapore General Hospital, \\ Singapore, Singapore
}

Background: The influence of being small for gestational age (SGA) on outcome of premature infants is controversial.

Aims: To compare early, two-year and five-year growth and neurodevelopmental outcomes of very low birthweight (VLBW) SGA infants with those appropriate for gestational age (AGA).

Methods: A retrospective, case-control study matched for gestational age was performed. Early outcome data were retrieved from medical records. Long term follow-up consisted of clinical examination and formal psychological assessments using the Bayley Scales of Infant Development (BSID) or Wechsler Preschool and Primary Scale of Intelligence (WPPSI).

Results: For the period 2003-2006, 33 SGA infants were matched with 66AGA infants. SGA infants were associated with maternal hypertensive disorder, perinatal foetal distress, lower 1-minute apgar scores and higher CRIB and CRIB II scores. SGA infants had higher rates of pulmonary haemorrhage, hypoglycaemia and thrombocytopaenia. There was no difference in mean BSID scores at 2 years, or mean full scale IQ scores at 5 years, although SGA infants tended to have higher verbal IQ scores and lower performance IQ scores. There was no difference in the incidence of neurodevelopmental impairment in both groups. Weight and head circumference at 2 years were significantly smaller for the SGA infants, however, at 5 years there were no growth differences observed between the 2 groups. 